\title{
An Adaptive Diagonal Loading Covariance Matrix Estimator in Spatially Heterogeneous Sea Clutter
}

\author{
Yanling Shi, Xiaoyan Xie \\ College of Telecommunications \& Information Engineering \\ Nanjing University of Posts and Telecommunications \\ Nanjing 210003, China \\ Email:ylshi@njupt.edu.cn m18625156782@163.com
}

Keywords: diagonal loading; covariance matrix estimator; heterogeneous sea clutter; radar target detection

\begin{abstract}
The most typical method to estimate the covariance matrix of sea clutter by secondary samples is the sample covariance matrix (SCM), implying secondary samples with the same statistical property. The hypothesis established or not depends on whether or not sea clutter is spatially homogeneous. In order to get rid of the hypothesis on secondary samples, a novel covariance matrix estimation algorithm based on adaptive diagonal loading technique is presented. The loading coefficient is a factor to measure the statistical consistency of secondary samples. Experimental results show that, to detect the distributed targets in real sea clutter by generalized likelihood ratio detector (GLRT), the proposed estimator is robust to jamming and the performance improves in different range resolution sea clutter, especially as high as $15 \mathrm{~dB}$ in $3 \mathrm{~m}$ range resolution.
\end{abstract}

\section{Introduction}

It is well known that sample covariance matrix (SCM) estimator is the maximum likelihood estimate of covariance matrix in Gaussian white noise [1]. It is simple in operation and small in computation. For target detection in sea clutter, SCM estimator works well in the low range resolution where sea clutter can be modelled as complex Gaussian random process. Secondary samples are with the same statistical properties, such as share the same covariance matrix. However, with the improvement of radar range resolution, sea clutter in the range cell is the vector superposition of only a few scatterers. Sea clutter is spatially heterogeneous [2], which is mainly reflected in the inconsistency of covariance matrix of sea clutter. For the high range resolution, due to the statistical inconsistency of secondary samples, SCM estimator has a large error in estimating covariance matrix, which will lead to a performance loss of detector.

In this letter, an adaptive covariance matrix estimator based on the diagonal loading (DL) is proposed. It combines SCM with the unit matrix adaptively, where the coefficient of combination changes with the statistical property of secondary samples adaptively. Experimental results show that, to detect the distributed target in real sea clutter by generalized likelihood ratio test (GLRT) detector [3], the proposed estimator is robust to jamming, and its performance has a significant improvement in high range radar resolution sea clutter, compared with SCM.

\section{Problem Description}

Let $N$-dimensional complex vector $\mathbf{z}_{r}, \boldsymbol{c}_{r}, \boldsymbol{p}$ be the received echoes, sea clutter and Doppler steering vector, $r=1,2, \cdots, L$, where $L$ is the number of range cells that target presences. $\alpha_{r}$, $r=1,2, \cdots, L$, are unknown constants relevant to target radar cross section. $\mathbf{z}_{r}, r=L+1, \cdots, L+K$, are secondary samples. Target detection in sea clutter is a conventional binary hypothesis testing, defined as 


$$
\left\{\begin{array}{l}
H_{0}: \mathbf{z}_{r}=\boldsymbol{c}_{r}, \quad r=1,2, \cdots, L, L+1, \cdots, L+K \\
H_{1}:\left\{\begin{array}{c}
\mathbf{z}_{r}=\alpha_{r} \boldsymbol{p}+\boldsymbol{c}_{r}, \quad r=1,2, \cdots, L \\
\mathbf{z}_{r}=\boldsymbol{c}_{r}, \quad r=L+1, \cdots, L+K
\end{array}\right.
\end{array}\right.
$$

For the distributed target, the GLRT detector [3] is

$$
t=-N \sum_{r=1}^{L} \ln \left(1-\frac{\left|\boldsymbol{p}^{\mathrm{H}} \hat{\boldsymbol{M}}^{-1} \mathbf{z}_{r}\right|^{2}}{\left(\boldsymbol{p}^{\mathrm{H}} \hat{\boldsymbol{M}}^{-1} \boldsymbol{p}\right)\left(\mathbf{z}_{r}^{\mathrm{H}} \hat{\boldsymbol{M}}^{-1} \mathbf{z}_{r}\right)}\right)
$$

where $\hat{\boldsymbol{M}}$ is the estimated temporal covariance matrix of the clutter vector $\boldsymbol{c}_{r}$. In the letter, the superscript $\mathrm{H}$ denotes conjugate transpose, and the superscript $\mathrm{T}$ denotes transpose.

\section{Proposed Estimator}

According to the statistical inconsistency of sea clutter in the high range resolution radar, a new estimator of covariance matrix of the clutter vector $\boldsymbol{c}_{r}$ is presented

where $\boldsymbol{M}^{*}$ is SCM, given by

$$
\hat{\boldsymbol{M}}=(1-\beta) \boldsymbol{M}^{*}+\beta \boldsymbol{I}
$$

$$
\boldsymbol{M}^{*}=\frac{1}{K} \sum_{r=L+1}^{L+K} \mathbf{z}_{r} \mathbf{z}_{r}^{\mathrm{H}}
$$

and $\boldsymbol{I}$ is an unit matrix. The weighted coefficient $\beta$ is a factor to measure the statistical consistency of secondary samples, calculated as follows

$$
\beta=\frac{\sum_{r=L+1}^{L+K}\left\|\mathbf{z}_{r} \mathbf{z}_{r}^{\mathrm{H}}-\boldsymbol{M}^{*}\right\|^{2}}{K^{2}\left\|\boldsymbol{M}^{*}\right\|^{2}}
$$

Symbol $\|\boldsymbol{A}\|=\sqrt{\operatorname{tr}\left(\boldsymbol{A} \boldsymbol{A}^{\mathrm{H}}\right)}$. Formula (3) involves $\mathrm{O}(2 K+1) N^{2}$ floating-point operations. In addition, it is easy to prove $0 \leq \beta \leq 1$.

The factor $\beta$ reflects the statistical consistency of covariance matrix of secondary samples $\mathbf{z}_{r}, r=L+1, \cdots, L+K$, the numerator of factor $\beta$ is equivalent to the variance of exterior product of $\boldsymbol{z}_{r}$, reflecting the deviation of exterior product from the mean of SCM. If the degree of deviation is large, the exterior product matrix is instable, and there is a bad statistical consistency of covariance matrix of $z_{r} \mathrm{~s}$ in different range cells, which is corresponding to heterogeneous sea clutter background. Thus, SCM will obviously bring in estimation errors. Hence, it is necessary to use $\beta$ to modify the estimation results. And, vice versa.

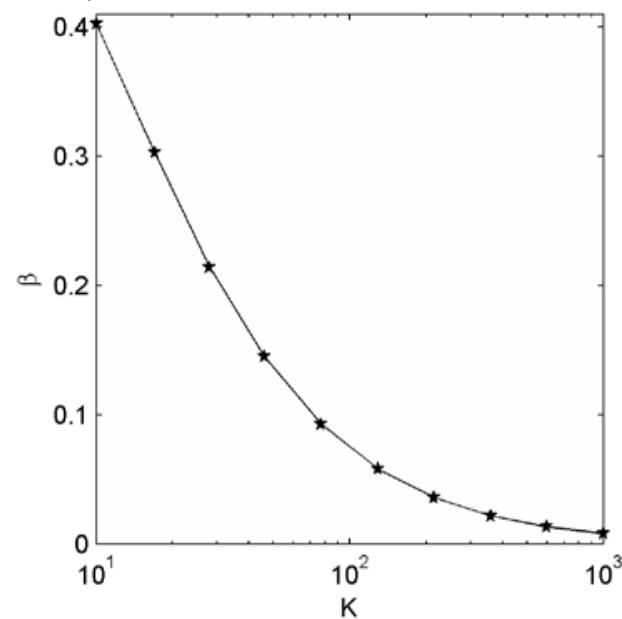

Fig. 1 The change of $\beta$ with the increasing $K$

In addition, because $\beta$ reflects the degree of deviation between two matrixes, considered the change of $\beta$ with the number of secondary samples, the graph of $\beta$ versus $K$ is shown in Fig 1 by 
using the simulation data, where $\mathbf{z}_{r} \sim C N(\mathbf{0}, \mathbf{I}), N=8, L=0$. The simulation was conducted with 50 Monte-Carlo runs. From Fig 1 , the curve shows the fall in $\beta$ with increasing $K$. The larger $K$, the smaller $\beta$, and tend to 0 eventually, namely $\lim _{K \rightarrow \infty} \beta=0$. When the clutter is spatially homogeneous for a larger $K$, corresponding to an abundant of secondary samples available, $\hat{\boldsymbol{M}}$ tends to $\boldsymbol{M}^{*}$. Thus, the covariance matrix can almost be estimated by SCM accurately. So, it makes no sense to calculate $\beta$ any more. When the number of the secondary sample is small, a deviation will be brought in by SCM. Therefore, it is indispensable to introduce the parameter $\beta$ to revise the estimation results. The conclusion can be reached that the proposed estimator is suitable for the spatially heterogeneous and/or a small number of secondary samples available.

Let the eigendecomposition of $\boldsymbol{M}^{*}$ be

$$
\boldsymbol{M}^{*}=\boldsymbol{U} \boldsymbol{\Lambda} \boldsymbol{U}^{H}
$$

where $\boldsymbol{U}$ is the clutter subspace matrix, $\boldsymbol{\Lambda}$ is diagonal matrix composed of the eigenvalues of clutter,

$$
\boldsymbol{\Lambda}=\operatorname{diag}\left(\lambda_{1}, \lambda_{2}, \cdots, \lambda_{N}\right) \quad \lambda_{1}>\lambda_{2}>\cdots>\lambda_{N}
$$

Substituting (6) into (2), it can be shown that

$$
\hat{\boldsymbol{M}}=(1-\beta) \boldsymbol{U}\left[\boldsymbol{\Lambda}+\frac{\beta}{1-\beta} \boldsymbol{I}\right] \boldsymbol{U}^{\mathrm{H}}
$$

The eigenvalues of $\hat{\boldsymbol{M}}$ are $(1-\beta) \lambda_{n}+\beta, n=1,2, \cdots, N$. The inverse matrix of $\hat{\boldsymbol{M}}$ is,

$$
\hat{\boldsymbol{M}}^{-1}=(1-\beta)^{-1} \boldsymbol{U}\left[\boldsymbol{\Lambda}+\frac{\beta}{1-\beta} \boldsymbol{I}\right]^{-1} \boldsymbol{U}^{\mathrm{H}}
$$

Considering the influence of clutter power, we normalize the eigenvalue by $\lambda_{1}$. Thus

$$
\begin{aligned}
\hat{\boldsymbol{M}}^{-1} & =(1-\beta)^{-1} \boldsymbol{U}\left[\boldsymbol{\Lambda} / \lambda_{1}+\frac{\beta}{1-\beta} \boldsymbol{I}\right]^{-1} \boldsymbol{U}^{\mathrm{H}} \\
& =\frac{\lambda_{1}}{1-\beta} \boldsymbol{U}\left[\boldsymbol{\Lambda}+\frac{\lambda_{1} \beta}{1-\beta} \boldsymbol{I}\right]^{-1} \boldsymbol{U}^{\mathrm{H}}
\end{aligned}
$$

Substituting (10) into (2), the GLRT detector is related with the loading coefficient $\lambda_{1} \beta /(1-\beta)$.

From formula (8), $\hat{\boldsymbol{M}}$ has a similar form to the DL algorithm in space-time adaptive processing (STAP) [4] which compresses eigenvalues of noise, weakens the adaptive capacity to small jammings, with the aim of decreasing the system sensitivity. The loading coefficient in STAP is generally set by experience, which may deviate away from an actual environment and bring in an error. As to guarantee the invertibility of the covariance matrix with small number of secondary samples, the DL algorithm is used to estimate the covariance matrix of clutter, where the loading coefficient is 2-3 times of the noise power [5]. So the loading coefficient is also an empirical value. In the letter, $\beta$ is adaptive with the statistical consistency of secondary samples. The loading coefficient is set with an actual condition, no longer an experience value.

For spatially heterogeneous sea clutter in high radar range resolution, the covariance matrix of sea clutter varies with the spatial secondary samples [6]. Secondary samples do not necessarily share the same covariance matrix. More precisely, we assume that the secondary samples can be clustered into $M$ groups, each one of them containing $L_{m}$ snapshots and sharing the same covariance matrix. The particular case $M=1$ corresponds to the scenario where sea clutter is spatially homogeneous. The extreme case $L_{m}=1$ for $m=1, \cdots, M$ would correspond to a situation where all secondary samples would have a different covariance matrix. Let $\boldsymbol{L}=\left[L_{1}, \cdots, L_{m}, \cdots, L_{M}\right]^{\mathrm{T}}$, $\sum_{m=1}^{M} L_{m}=K$. Regroup the secondary samples as $\boldsymbol{Z}=\left[\mathbf{z}_{L+1}, \cdots, \mathbf{z}_{r}, \cdots, \mathbf{z}_{L+K}\right]=\left[\boldsymbol{Z}_{1}, \cdots, \boldsymbol{Z}_{m}, \cdots, \boldsymbol{Z}_{M}\right]$, where $\mathbf{z}_{r} \in \mathrm{C}^{N \times 1}$ and $\boldsymbol{Z}_{m} \in \mathrm{C}^{N \times L_{m}}$. The SCM based on $\boldsymbol{Z}_{m}$ is denoted as

$$
\boldsymbol{M}_{m}^{*}=\frac{1}{L_{m}} \mathbf{Z}_{m} \mathbf{Z}_{m}^{\mathrm{H}}
$$


Similarly, based on (3), the estimation of covariance matrix $\hat{\boldsymbol{M}}_{m}$ is

$$
\begin{aligned}
\hat{\boldsymbol{M}}_{m} & =\left(1-\beta_{m}\right) \boldsymbol{M}_{m}{ }^{*}+\beta_{m} \boldsymbol{I} \\
& =\boldsymbol{U}_{m}\left[\left(1-\beta_{m}\right) \boldsymbol{\Lambda}_{m}+\beta_{m} \boldsymbol{I}\right] \boldsymbol{U}_{m}^{\mathrm{H}}
\end{aligned}
$$

where $\boldsymbol{U}_{m}, \boldsymbol{\Lambda}_{m}$ and $\beta_{m}$ are the eigenvector matrix, eigenvalue matrix and weighted coefficient of $\boldsymbol{M}_{m}{ }^{*}$, respectively.

Normalize the eigenvalue by $\lambda_{m, 1}$,

$$
\hat{\boldsymbol{M}}_{m}=\frac{1-\beta_{m}}{\lambda_{m, 1}} \boldsymbol{U}_{m}\left[\boldsymbol{\Lambda}_{m}+\frac{\lambda_{m, 1} \beta_{m}}{1-\beta_{m}} \boldsymbol{I}\right] \boldsymbol{U}_{m}^{\mathrm{H}}
$$

where $\lambda_{m, 1}$ is the maximum eigenvalue of $\boldsymbol{\Lambda}_{m}$.

So, the covariance matrix estimator of $\mathbf{Z}$ is given by

$$
\begin{aligned}
\hat{\boldsymbol{M}} & =\frac{1}{M} \sum_{m=1}^{M} \hat{\boldsymbol{M}}_{m} \\
& =\frac{1}{M} \sum_{m=1}^{M} \frac{\left(1-\beta_{m}\right)}{\lambda_{m, 1}} \boldsymbol{U}_{m}\left[\boldsymbol{\Lambda}_{m}+\frac{\lambda_{m, 1} \beta_{m}}{1-\beta_{m}} \boldsymbol{I}\right] \boldsymbol{U}_{m}^{\mathrm{H}}
\end{aligned}
$$

Substituting (14) into (2), the GLRT detector is related not only with the loading coefficient $\lambda_{m, 1} \beta_{m} /\left(1-\beta_{m}\right)$ but also with $\left(1-\beta_{m}\right) / \lambda_{m, 1}$.

\section{Experimental Work}

We now illustrate the performance of the various estimation schemes introduced in the previous sections. The following experiment uses the real sea clutter data collected by IPIX radar (http://soma.mcmaster.ca/ipix.php). The file is 19980223-170435 with the range resolution 15m. The synthetic target returns are expressed as:

$$
S_{r}(n)=a_{r} \exp \left(\mathrm{i}\left(2 \pi n f_{d} T+\varphi\right)\right), n=1,2, \cdots, N, r=1, \cdots, L
$$

where $f_{d}$ is the target Doppler frequency, $T$ is the coherent processing interval, the initial phase $\varphi$ is a uniform distributed random number in $[-\pi, \pi]$ in trials, and $a_{r}$ is a constant relevant to radar cross section of target used to adjust signal-to-clutter ratio (SCR), defined by

$$
S C R=10 \log _{10}\left(L a_{r}^{2} / P_{c}\right)
$$

where $P_{c}$ is the average power of sea clutter time series. $a_{1}=a_{2}=\cdots=a_{L}$. The false alarm probability is taken as 0.001 . The parameters are set as $L=2, N=4, K=28, f_{d}=-100 \mathrm{~Hz}$. We vary the number of groups $M$. More specifically, we consider four cases with $M=1$ (correspond to formula (8)), $M=2$ and $M=4$, respectively. For $M=4$, we consider two cases: $L_{1}=\cdots=L_{m}=\cdots=L_{M}$ and different $L_{m}$ in each group. This means that there exist 1,2 or 4 different covariance matrices among the secondary samples. The four cases are defined by

case 1: $\mathbf{L}=28$; case 2: $\boldsymbol{L}=[14,14]^{\mathrm{T}}$; case $3: \mathbf{L}=[7,7,7,7]^{\mathrm{T}}$; case $4: \mathbf{L}=[6,8,8,6]^{\mathrm{T}}$.

Fig 2 gives the detection performance of SCM and proposed estimators in four cases. For detecting the distributed target in real sea clutter by GLRT, the proposed estimator obviously outperforms SCM.

In order to measure the robust of the proposed estimator, we add a jamming at the first range cell. The jamming is

$$
I(n)=B \exp \left(\mathrm{i}\left(2 \pi n f_{i} T+\varphi_{i}\right)\right)
$$

where $f_{i}=-400 \mathrm{~Hz}, \varphi_{i}$ is a uniform distributed random number in $[-\pi, \pi]$ in trials, and $B$ is adjust as interference-to-clutter ratio (ICR), defined by

$$
I C R=10 \log _{10}\left(B^{2} / P_{c}\right)
$$


Fig 3 gives the detection performance of SCM and proposed estimators in four cases with ICR = $10 \mathrm{~dB}$. Compared fig. 2 with fig. 3, the results presented prove grouping is of advantage in improving anti-jamming capability.

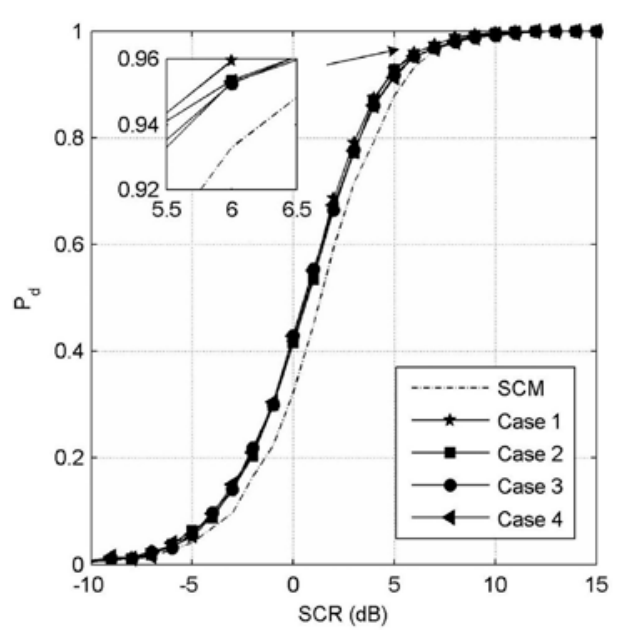

Fig. 2 Pd versus SCR of GLRT detector with different covariance matrix estimators without interference.

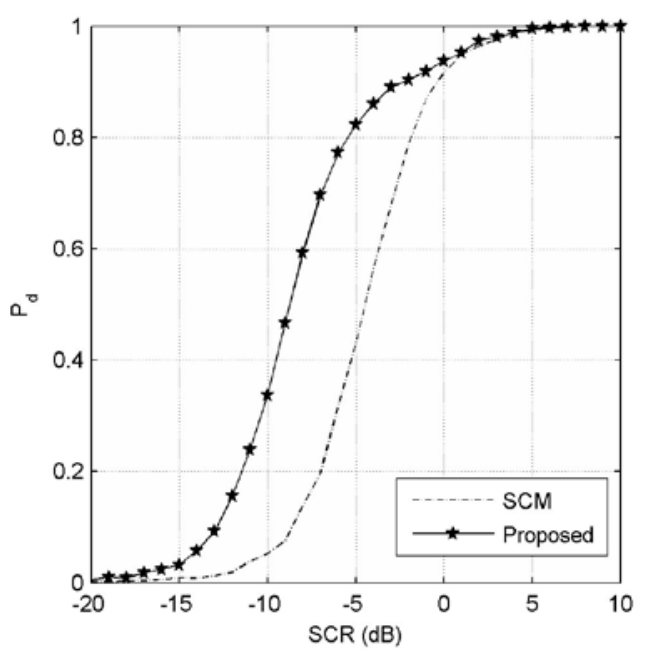

(a)

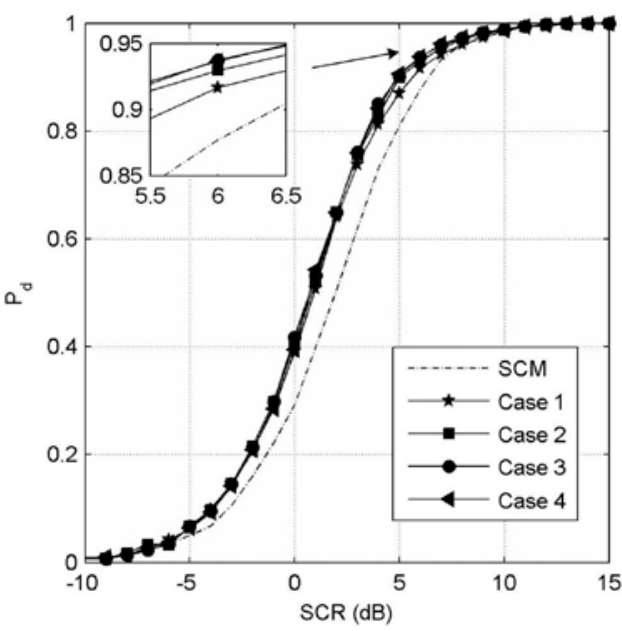

Fig. 3 Pd versus SCR of GLRT detector with different covariance matrix estimators with ICR = $10 \mathrm{~dB}$.

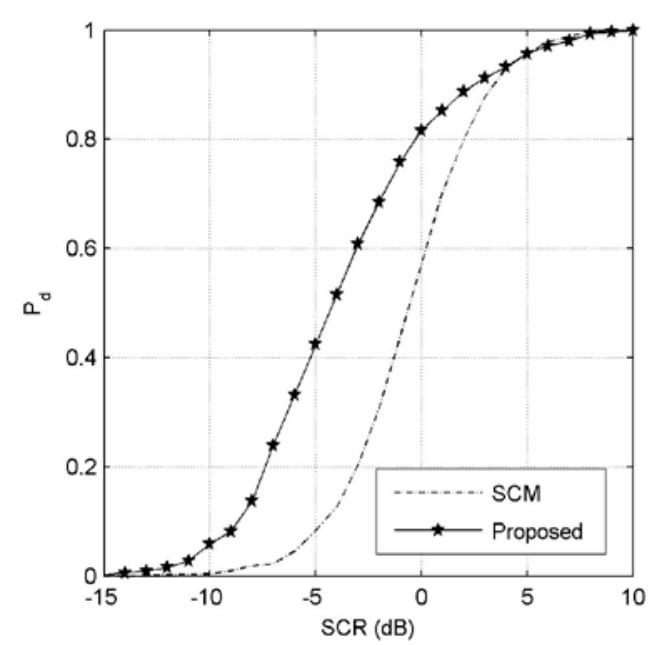

(b)

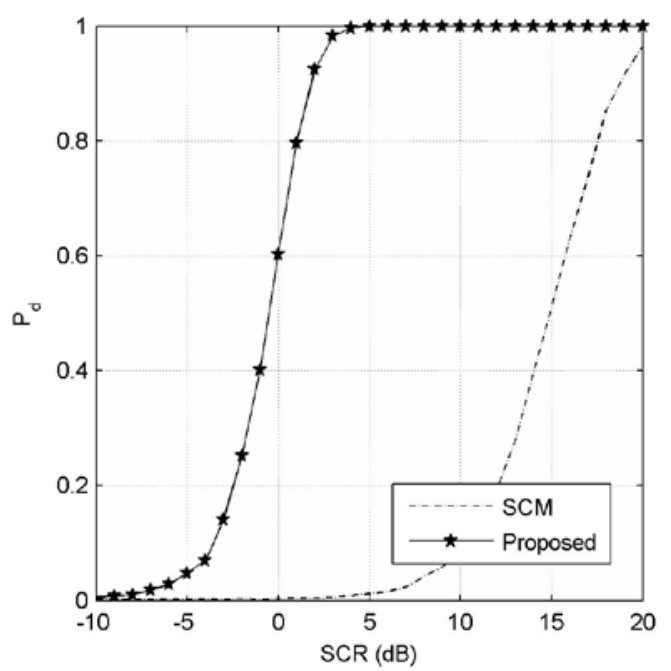

(c)

Fig. 4 Pd versus SCR of GLRT detector with proposed estimator and SCM with the range resolution (a) 15m, (b) $9 \mathrm{~m}$, (c) $3 \mathrm{~m}$. 
In following, we analyze the performance of spatially heterogeneous sea clutter in different range resolution. Suppose that the length of target is $30 \mathrm{~m}$ and $N=18, M=1$. The parameters are $K=28$ and $L=2$ for $19980223-170435$ ( the range resolution is $15 \mathrm{~m}$ ), $K=26$ and $L=4$ for 19980223173950( the range resolution is $9 \mathrm{~m}$ ), $K=20$ and $L=10$ for $19980223-172410$ ( the range resolution is $3 \mathrm{~m})$, respectively.

Fig. 4 gives the detection performance of SCM and proposed estimator. The proposed estimator performs even better than SCM with the higher range resolution.

\section{Conclusion}

In order to overcome the statistical inconsistency of secondary samples in the high range resolution, a robust covariance matrix estimator based on the adaptive DL is proposed. It is suitable for spatially heterogeneous and/or a small number of secondary samples available. The experimental results show that, to detect the distributed target in real sea clutter by GLRT, the proposed estimator is robust to jamming and the performance improves in different range resolution sea clutter, especially as high as $15 \mathrm{~dB}$ in $3 \mathrm{~m}$ range resolution.

\section{Acknowledgment}

This work was supported by National Natural Science Fund (61201325) and Jiangsu Government Scholarship for Overseas Studies.

\section{References}

[1]M. Rangaswamy, "Statistical analysis of the nonhomogeneity detector for non-Gaussian interference backgrounds,” IEEE Trans. Signal Processing, vol. 53, no.6, pp. 2101-2111, 2005.

[2]M. Greco, P. Stinco, F. Gini, "Impact of sea clutter nonstationarity on disturbance covariance matrix estimation and CFAR detector performance,” IEEE Trans. Aerosp. Electron. Syst, vol. 46, no.3, pp. 1502-1513, September, 2010.

[3]X. F. Shuai, L. J, Kong and J. Y. Yang, "Performance analysis of GLRT-based adaptive detector for distributed targets in compound-Gaussian clutter," Signal processing, Vol. 90, pp. 16-23, 2010.

[4]G. Ginolhac, P. Forster, J. P. Ovarlez, et al. "Spatio-temporal adaptive detector in nonhomogeneous and low-rank clutter," IEEE International Conference on Acoustics, Speech and Signal Processing, pp. 2045-2048, 2009.

[5]I. A.Yuri, A. J. Ben, K. S. Nicholas, "Sample-Deficient adaptive detection: adaptive scalar thresholding versus CFAR detector performance," IEEE Trans. on Aerospace and Electronic Systems, vol. 46, no. 1, pp. 32-46, January, 2010.

[6]B. Olivier, B. Stéphanie, T. Jean-Yves, "Covariance matrix estimation with heterogeneous samples,” IEEE Trans. Signal Processing, vol.56, no. 3, pp. 909-920, 2008. 